\title{
Associations between Electrophysiological Evidence of Reward and Punishment-Based Learning and Psychotic Experiences and Social Anhedonia in At-Risk Groups
}

\author{
Nicole R Karcher', Bruce D Bartholow', Elizabeth A Martin² and John G Kerns*, \\ 'Department of Psychological Sciences, University of Missouri, Columbia, MO, USA; ${ }^{2}$ Department of Psychology and Social Behavior, University of \\ California, Irvine, CA, USA
}

\begin{abstract}
Both positive psychotic symptoms and anhedonia are associated with striatal functioning, but few studies have linked risk for psychotic disorders to a neural measure evoked during a striatal dopamine-related reward and punishment-based learning task, such as a reversal learning task (RLT; Cools et al, 2009). The feedback-related negativity (FRN) is a neural response that in part reflects striatal dopamine functioning. We recorded EEG during the RLT in three groups: (a) people with psychotic experiences (PE; $n=20$ ) at increased risk for psychotic disorders; (b) people with extremely elevated social anhedonia (SocAnh; $n=22$ ); and (c) controls $(n=20)$. Behaviorally, consistent with increased striatal dopamine, the PE group exhibited better behavioral learning (ie, faster responses) after unexpected reward than after unexpected punishment. Moreover, although the control and SocAnh groups showed a larger FRN to punishment than reward, the PE group showed similar FRNs to punishment and reward, with a numerically larger FRN to reward than punishment (with similar results on these trials also found for a P3a component). These results are among the first to link a neural response evoked by a reward and punishment-based learning task specifically with elevated psychosis risk.

Neuropsychopharmacology (2017) 42, 925-932; doi:I0.1038/npp.2016.192; published online 12 October 2016
\end{abstract}

In psychotic disorders, there is evidence that the striatum might be involved in both positive (eg, Howes and Kapur, 2009) and negative symptoms (eg, Juckel et al, 2006). Given confounds often present in patient research (eg, medication effects; Demjaha et al, 2012), it is also important to examine associations between disorder risk and striatal functioning. However, very few studies have linked either psychotic experiences (ie, psychotic-like experiences that predict increased risk for psychotic disorder; Chapman et al, 1994; Kaymaz et al, 2012) or anhedonia in at-risk populations to a neural response evoked by a striatal dopamine-related behavioral task. Moreover, in at-risk populations, there is only limited evidence of impairments in behavioral measures that are thought to be related to striatal functioning. Arguably, the best existing evidence is that first-degree relatives of people with schizophrenia exhibit impairments on striatal-related behavioral measures (ie, on a probabilistic category learning task; eg, Wagshal et al, 2012; Weickert et al, 2010). However, it is not clear from this work whether these behavioral impairments are related to risk for positive psychotic symptoms or risk for negative symptoms

\footnotetext{
*Correspondence: Dr JG Kerns, Department of Psychological Sciences, University of Missouri, 214 McAlester Hall, Columbia, MO 6521 I, USA, Tel: + I 573882 8846, Fax: + 573882 77।0,

E-mail: kerns@@missouri.edu

Received I April 2016; revised 23 August 2016; accepted I September 2016; accepted article preview online 15 September 2016
}

(or neither). Here we examined whether either psychotic experiences (PE) or social anhedonia (SocAnh) in at-risk groups is associated with behavioral and neural measures of reward and punishment-based learning.

A neural measure related to striatal dopamine is the feedback-related negativity (FRN), an event-related potential (ERP) component occurring $\sim 250 \mathrm{~ms}$ following performance feedback appearing most robustly in frontocentral electrodes (eg, Carlson et al, 2011). The FRN is typically larger (ie, more negative) for unexpected negative than positive feedback (eg, Proudfit, 2015). Hence, the FRN is often measured as the difference between negative $v s$ positive feedback (eg, Nieuwenhuis et al, 2005). The FRN has consistently been linked to activation in the striatum (eg, Carlson et al, 2011; Becker et al, 2014; although other regions are also involved in its generation; eg, Baker and Holroyd, 2011).

In particular, the FRN is thought to be influenced by phasic changes in striatal dopamine (Becker et al, 2014; Walsh and Anderson, 2012). Consistent with this, administration of dopamine agonists has been found to increase the FRN specifically for correct positive feedback trials (Santesso et al, 2009). One interpretation of this result is that increased dopamine produces increased salience for positive feedback. This interpretation is consistent with evidence that the FRN is influenced by the salience of outcomes (eg, Pfabigan et al, 2015), that increased striatal dopamine is associated with heightened sensitivity to rewards and decreased sensitivity to punishments (Collins and Frank, 2014), and that evidence of 
increased sensitivity to rewards is associated with increased FRN for unexpected rewards (Smillie et al, 2011). The reversal learning task (RLT) developed by Cools et al (2009) involves periodic, unexpected positive and negative feedback, with increased striatal dopamine (either baseline levels or due to pharmacological manipulation) associated with behavioral evidence of better learning after unexpected reward relative to unexpected punishment (Cools et al, 2009; van der Schaaf et al, 2014). In the current study, behavioral performance on the RLT, measured concurrently with the FRN, was used to index the extent to which abnormal reward and punishment-based learning is apparent in PE or SocAnh risk groups.

To our knowledge, no previous study has examined the FRN or the RLT and risk for schizophrenia-spectrum disorders. We expected that the results for $\mathrm{PE}$ would be consistent with abnormal reward and punishment-based learning (Karcher et al, 2015a). Behaviorally, we expected that PE would be associated with better learning from unexpected reward than from unexpected punishment and we also expected that PE would be associated with a relatively larger FRN for unexpected reward than for unexpected punishment. As far as SocAnh, based on possible deficits in processing rewards, we expected that SocAnh would be related to a relatively smaller FRN for unexpected reward than for unexpected punishment. Further, to examine whether any associations with the FRN were not due to group differences in the P3a, a component that partially overlaps with the FRN's scalp distribution that is known to be highly sensitive to novelty (Polich, 2007) and that follows the FRN in time, we also examined P3a amplitudes on unexpected feedback trials (ie, to examine whether what appears to be a larger FRN could actually reflect a smaller $\mathrm{P} 3 \mathrm{a})$.

\section{MATERIALS AND METHODS}

\section{Participants}

The PE group consisted of 20 people (55\% women; mean age $=18.45$ years $(\mathrm{SD}=0.60) ; 70 \%$ Caucasian $)$ who scored $>1.96$ SD above the same-sex mean on the Perceptual Aberration (Chapman et al, 1978) or Magical Ideation (Eckblad and Chapman, 1983) scales or had a summed, standardized score from the Perceptual Aberration and Magical Ideation (PerMag) scales > 3.0. People with extremely elevated PerMag scores have been found to be at increased risk for psychotic disorders (Chapman et al, 1994). Further, all $\mathrm{PE}$ participants in the current study also had current interview ratings $\geqslant 2$ (with 2 =presence of attenuated $\mathrm{PE}$ ) on both Unusual Thought Content/Delusional Ideation and Perceptual Abnormalities/Hallucinations subscales of the Structured Interview for Prodromal Syndromes (SIPS; Miller et al, 2003; with the SIPS having been used successfully to predict risk for future psychotic disorder; current inter-rater reliabilities for two raters $\geqslant 0.93$; in the current study, no participant scored a 6 on any of the domains of the SIPS, indicating no frank psychosis).

The SocAnh group contained 22 people $(65.2 \%$ women; mean age $=18.74$ years $(\mathrm{SD}=0.81) ; 65 \% \quad$ Caucasian $)$ who scored $>1.96 \mathrm{SD}$ above the same sex mean on the Revised SocAnh Scale (Eckblad et al, 1982). Extremely elevated SocAnh is rather selectively associated with the emergence of nonpsychotic schizophrenic-spectrum disorders' (Debbané et al, 2015). In order to more clearly distinguish psychosis risk from elevated SocAnh, to be recruited for this study the SocAnh (and control) groups had to be rated as never having PE $(\mathrm{ie},<2)$ on the SIPS.

There were 21 people in the control group (50\% women; mean age $=18.39$ years $(\mathrm{SD}=0.78) ; 79 \%$ Caucasian) who scored $<0.5$ SD below the mean on the Revised Social Anhedonia Scale, Perceptual Aberration Scale, and Magical Ideation Scale. All study participants were Introduction to Psychology students who participated for course credit after taking part in a separate behavioral testing session (Karcher et al, 2015b; see Supplementary Methods for recruitment details). In the current study, there were no significant differences between the three groups on any demographic variables (eg, sex, age, or ethnicity).

\section{Materials}

Reversal learning task. In general on reversal learning tasks, participants first learn that one stimulus is rewarded and that a second stimulus is punished. Subsequently, the associations with reward and punishment are switched and participants have to learn that the first stimulus is now punished and that the second stimulus is now rewarded, with this pattern repeating through a number of reversals. There are two trial types that are most important for this task: (1) unexpected reward trials, where a stimulus that was previously associated with punishment is now associated with reward; and (2) unexpected punishment trials, where a stimulus previously associated with reward is now associated with punishment. On each trial two horizontally adjacent stimuli were presented simultaneously, one face and one natural scene (eg, depicting a mountain range), one of which was highlighted with a yellow border. The participant predicted whether the highlighted stimulus would lead to a reward or a punishment, indicating their prediction by pressing one of two buttons on a response box (both stimuli remained on-screen until the participant responded; Cools et $a l, 2009)$. The participant's self-paced response was followed immediately by an outcome message, presented for $500 \mathrm{~ms}$ (and following the outcome message, there was an inter-trial interval that jittered between 1000 and $1500 \mathrm{~ms}$ ). Reward consisted of a green smiley face and the words 'You Win!'. Punishment consisted of a red sad face and the words 'You Lose'. Through trial and error, participants learned which image was associated with reward or with punishment (ie, expected reward and expected punishment trials; note that participants were not actually rewarded or punished, beyond receipt of this visual feedback). Reward and punishment associations would change after the participant got a random number (between 5 and 9) of consecutive trials correct (ie, unexpected reward and unexpected punishment trials; Cools et al, 2009). After a reversal/unexpected feedback trial, the same stimulus would also be highlighted on the very next trial. Following Cools et al, each subject performed one practice block followed by four additional blocks, each consisting of 120 trials (Cools et al, 2009). The practice block included one reversal, following which participants had to get 20 (not necessarily consecutive) trials correct before moving on to the experimental blocks, with this criterion imposed to ensure that participants were 
acclimated to the task (Cools et al, 2009; see Supplementary Methods for more details about the task).

We examined the FRN on these unexpected reversal trials that involve a change in reward $v s$ punishment stimulus mapping that then result in participants getting unexpected feedback that they have made an error, and we compared the FRN for unexpected reward trials $v s$ unexpected punishment trials. We examined behavioral learning from unexpected reward and unexpected punishment by examining reaction time (RT) and accuracy on the trial immediately following unexpected feedback. We computed Reward Sensitivity Differences Scores (for RT: unexpected punishment minus unexpected reward; for accuracy: unexpected reward minus unexpected punishment), with higher reward sensitivity difference scores indicating faster and more accurate learning after unexpected reward than after unexpected punishment. In addition, in order to examine whether results were specific to the unexpected reward and unexpected punishment trials, we also examined expected reward and expected punishment trials (ie, trials that did not involve a change in reward or punishment stimulus mappings).

\section{Electrophysiological Recording and Analysis}

Consistent with previous research, we examined the FRN as the average voltage deflection between 225 and $325 \mathrm{~ms}$ postoutcome/feedback (eg, Bismark et al, 2013; electrophysiological recording details can be found in the Supplementary Methods). The FRNs at the frontocentral electrodes (F3, FZ, F4, FC3, FCZ, and FC4) were examined for each condition since previous research indicates that the FRN is maximal at frontocentral locations (Holroyd et al, 2003). The FRN was maximal at the FCZ electrode. The P3a was measured as the average voltage deflection between 350 and $500 \mathrm{~ms}$ post-outcome/feedback at the FCZ and CZ electrodes (ie, where the P3a was maximal; Evans et al, 2013). The P3b was measured as the average voltage deflection between 500 and $600 \mathrm{~ms}$ post-outcome/ feedback at the PZ electrode, where the P3b was maximal.

\section{Procedure and Data Analyses}

After completion of the questionnaire measures and the SIPS (Karcher et al, 2015b), eligible participants were invited to participate in the current study. After electrode placement while in a soundproof recording chamber, participants practiced and completed the RLT (as well as another, unrelated task not reported here), presented using E-Prime software (Psychology Software Tools, Inc., 2006).

Reaction times $<200 \mathrm{~ms}$ and $>10000 \mathrm{~ms}$ were excluded from analyses. Cohen's $d$ is included as a measure of effect size, except for $F$ tests with numerator $\geqslant 2$ that report etasquared. Owing to excessive artifacts, EEG data from two participants (one PE and one control) had to be excluded from analyses.

The data were analyzed with mixed hierarchical linear modeling (HLM; Bates, 2010), which has several advantages over traditional repeated-measures analysis of variance for analyzing EEG data, particularly when sample sizes are modest (see Luck, 2005; Page-Gould, in press). The HLM approach does not assume the data meet the criterion of sphericity (ie, that the variances of the differences between all group combinations are equal), an assumption that is frequently violated in psychophysiological data (Jennings and Wood, 1976), and therefore corrections for this violation that can decrease statistical power (eg, Greenhouse-Geisser $p$-value adjustments) are not necessary. Further, the use of an intercept for each electrode within each subject also helps to reduce error variance estimates, which also helps to increase statistical power. Finally, since HLM does not use listwise deletion for missing data, this approach is more robust to missing data, such as allowing bad electrodes to be rejected on an individual subject basis (Gratton, 2007). All ERP analyses examined amplitude using a 3 (group: PE, SocAnh, control) $\times 2$ valence (reward, punishment) HLM with subject modeled as a random intercept.

\section{RESULTS}

\section{Behavioral Task Performance}

Reaction time. For trials immediately following unexpected feedback, in a trial type (unexpected reward, unexpected punishment) $\times$ group ANOVA, there was a main effect of trial type, $F(1,59)=5.33, p<0.05, d=0.60$ (Table 1 , Figure 1), with faster responses after unexpected reward $(M=867.02, \quad S D=218.00)$ than unexpected punishment $(M=912.51, \mathrm{SD}=239.14)$. There was also a main effect of group, $F(2,59)=4.05, p<0.05, \eta^{2}=0.120$, with the PE group slower overall after unexpected feedback $(M=1000.24$, $\mathrm{SD}=215.09) ; v s$ control group $(M=847.00, \mathrm{SD}=227.02)$, $t(38)=2.14, \quad p<0.05, d=0.68 ;$ vs SocAnh $(M=832.79$, $\mathrm{SD}=166.47), \quad t(40)=2.79, \quad p<0.01, \quad d=0.86$ (control vs SocAnh $p=0.82, d=-0.07)$.

Table I Means (and SD) for Reaction Time (RT) and Accuracy by Group

\begin{tabular}{|c|c|c|c|c|c|c|}
\hline & \multicolumn{3}{|c|}{ RT } & \multicolumn{3}{|c|}{ Accuracy } \\
\hline & PE & SocAnh & Control & PE & SocAnh & Control \\
\hline Unexpected punishment (UP) & 1061.4* (247.4) & $819.5(171.8)$ & $865.9(232.7)$ & $0.81(0.13)$ & $0.84(0.14)$ & $0.85(0.11)$ \\
\hline Reward Sensitivity Difference Score & |32.5* (|20.1) & $-26.6(183.3)$ & $37.8(176.7)$ & $0.01(0.16)$ & $-0.01(0.15)$ & $0.01(0.12)$ \\
\hline
\end{tabular}

Abbreviations: PE, Psychotic Experiences group; SocAnh, Social Anhedonia group.

$R T=R T$ for trials immediately following unexpected feedback. Reward Sensitivity Difference Score for RT $=$ UP - UR; for Accuracy $=$ UR - UP. Asterisks indicate that the PE group differed significantly from the other two groups (note that for the Reward Sensitivity Difference Score for PE vs Controls, $p=0.06$ ). 


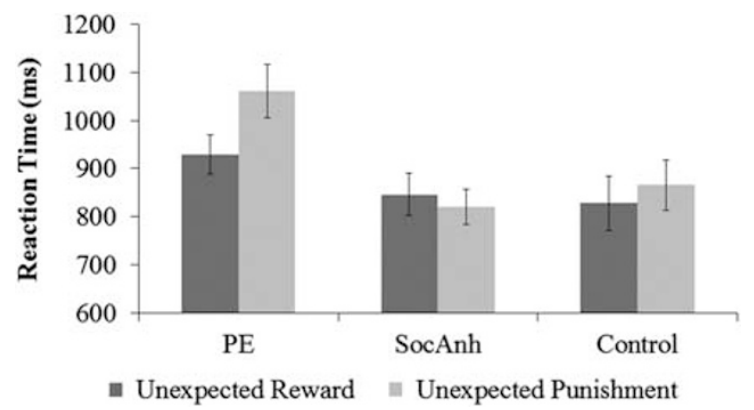

Figure I Better learning after unexpected reward than punishment on the Reversal Learning Task in the psychotic experiences group (PE). Reaction times are for the trial after the unexpected reward and the unexpected punishment trials. Error bars reflect SE.

Most importantly, there was a predicted trial type $\mathrm{X}$ group interaction, $F(2,59)=5.00, p<0.05, \eta^{2}=0.144$. Both control and SocAnh groups did not differ by unexpected feedback type; control $p=0.35, d=0.16$; SocAnh $p<0.50, d=-0.14$. In contrast, the PE group was faster for unexpected reward than for unexpected punishment, $t(18)=4.93, p<0.001, d=1.27$. Further, the difference in RT between unexpected reward and unexpected punishment (ie, the Reward Sensitivity Difference Score) was larger in the PE group $(M=127.19, \mathrm{SD}=120.96)$ compared with the SocAnh group $(M=-26.56, \mathrm{SD}=183.25)$, $t(40)=3.29, p<0.005, d=1.04$; the difference was marginally larger in the $\mathrm{PE}$ group compared with the control group $(M=37.76, \mathrm{SD}=176.69), t(38)=1.98, p=0.06, d=0.63$ (control and SocAnh groups did not differ, $p=0.26, d=0.37$ ). Hence, as expected, PE appeared to be associated behaviorally with increased sensitivity to reward relative to punishment. In a comparable analysis of accuracy, there were no significant main effects or interaction $(F s<1)$.

\section{FRN Amplitude}

In the HLM on FRN amplitude, the effects of valence $(p=0.13$, $d=-0.40)$ and group $(p=0.85, d=0.05)$ were not significant. However, as expected, there was a significant Valence $\times$ Group interaction, $t(675)=3.51, p<0.0005, d=0.91$ (Table 2 and Figure 2). Both control and SocAnh groups exhibited the typical pattern of larger (ie, more negative) FRN to unexpected punishment feedback than to unexpected reward feedback; control $t(213.84)=3.59, \quad p<0.001, \quad d=1.65 ; \quad$ SocAnh $t(235.95)=4.37, p<0.001, d=1.91$. In contrast, the PE group generally showed similar FRNs to unexpected punishment and unexpected reward, $t(213.99)=-1.15, p=0.25, d=-0.53$; if anything, unexpected reward elicited numerically larger FRNs than unexpected punishment in the PE group (Figure 2d).

Additional comparisons showed that the magnitude of the FRN to unexpected punishment did not differ across the groups, $F(2,59)=0.29, p=0.75, \eta^{2}=0.00$. In contrast, the FRN elicited by unexpected reward feedback differed significantly across the groups, $F(2,59)=5.93, p<0.005, \eta^{2}=0.03$, with the $\mathrm{PE}$ group showing a larger FRN in comparison with the other two groups (Figure $2 b$ ).

\section{Additional Analyses}

We performed several additional analyses to examine whether results for the PE group were specific to unexpected
Table 2 Feedback Related Negativity (FRN) Amplitude Means (and SD) for Unexpected Reward and Unexpected Punishment for each of the Groups

\begin{tabular}{|c|c|c|c|}
\hline \multirow[t]{2}{*}{ Condition } & \multicolumn{3}{|c|}{ Group } \\
\hline & PE & SocAnh & Control \\
\hline Unexpected punishment (UP) & $4.76(5.98)$ & $4.52(4.17)$ & $5.03(4.62)$ \\
\hline Unexpected reward (UR) & $4.38(5.6 \mathrm{I})$ & $6.36(3.78)$ & $6.61(5.81)$ \\
\hline $\begin{array}{l}\text { Reward Sensitivity Difference } \\
\text { Score (UP - UR) }\end{array}$ & $0.38(2.82)^{*}$ & $-1.84(4.67)$ & $-1.58(4.83)$ \\
\hline
\end{tabular}

Abbreviations: PE, psychotic experiences group; SocAnh, Social

Anhedonia group.

Smaller (ie, less positive/more negative) values = larger FRN. Asterisk indicates that the PE group differed significantly from the other two groups.

feedback trials and whether they were specific to the FRN (full results reported in the Supplementary Results Section). First, we examined behavioral performance and the FRN after correct expected feedback trials. Here there were no significant behavioral or FRN effects for group. Hence, significant FRN group differences for PE $v s$ the other two groups were specific to unexpected feedback trials.

Next we examined whether the significant group differences for the FRN reported previously could have reflected differences in the P3a (ie, whether what appears to be a larger FRN is actually due to a smaller P3a in frontocentral electrodes). Here, just as for the FRN, there was a significant Valence $\times$ Group interaction, $t(180.77)=-2.91, p<0.005$, $d=-0.76$ (Table 3). Both control and SocAnh groups exhibited non-significantly larger P3a for unexpected punishment than unexpected reward trials; controls $p=0.19$, $d=-0.61$; SocAnh $p=0.65, d=-0.20$ (with these groups not differing from each other, $p=0.48, d=-0.22$ ). In contrast, the PE group showed a significantly larger P3a for unexpected reward trials than unexpected punishment trials, $t(56.51)=2.52, p<0.05, d=1.16$, and the P3a to unexpected reward feedback was significantly larger in the PE group compared with both the control, $t(115.54)=-2.79, p<0.01$, $d=-0.91$ and SocAnh groups, $t(121.52)=-2.32, p<0.05$, $d=-0.73$. Hence, just as for the FRN, the PE group exhibited a larger P3a for unexpected reward than unexpected punishment, and therefore it is clear that the association between the FRN and PE cannot be accounted for by a reduction in the P3a.

\section{DISCUSSION}

Very few studies have linked either PE or anhedonia in atrisk populations to a neural response evoked by a reward and punishment-based learning task. The current research provides novel evidence that people with $\mathrm{PE}$ exhibit behavioral and neural deficits consistent with abnormal reward and punishment-based learning. In contrast to psychosis risk, the current results for SocAnh were quite different from PE. Hence, this suggests that in the current study reward and punishment-based learning dysfunction was specific to PE and therefore cannot be easily attributable to a general risk for schizophrenia-spectrum disorders. These 
a

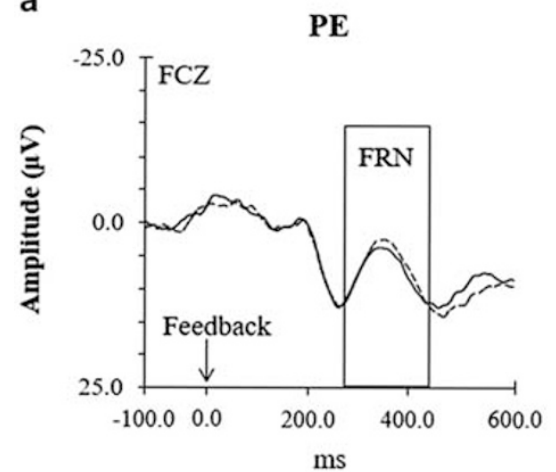

SocAnh

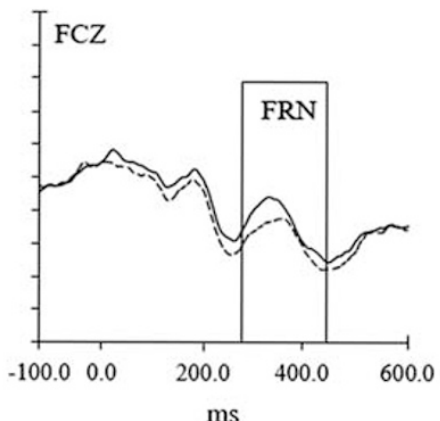

Control

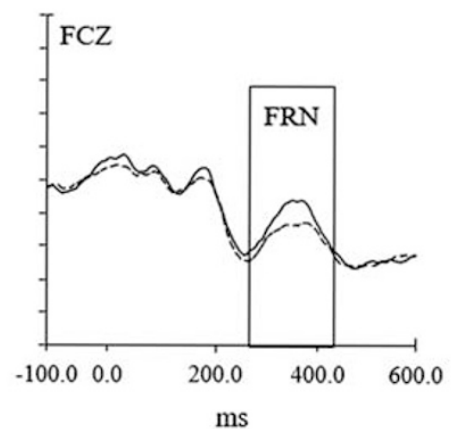

---. Unexpected Reward (UR)

Unexpected Punishment (UP)

b

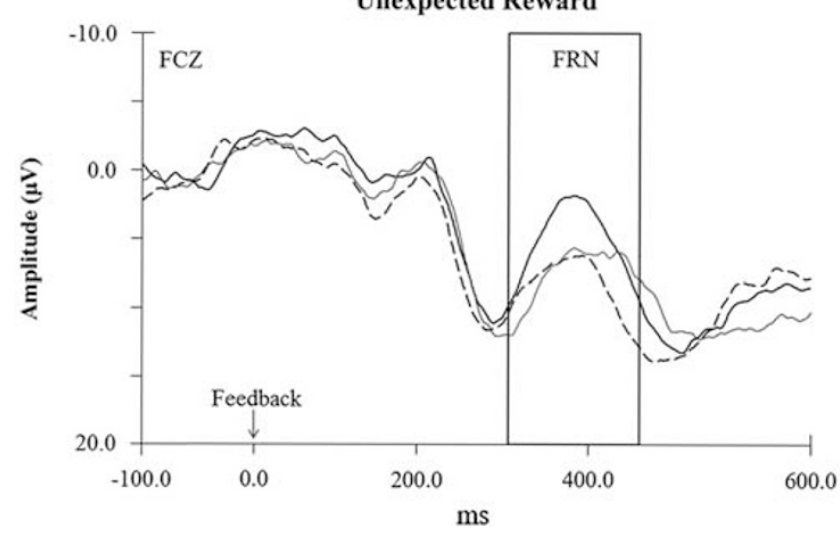

C

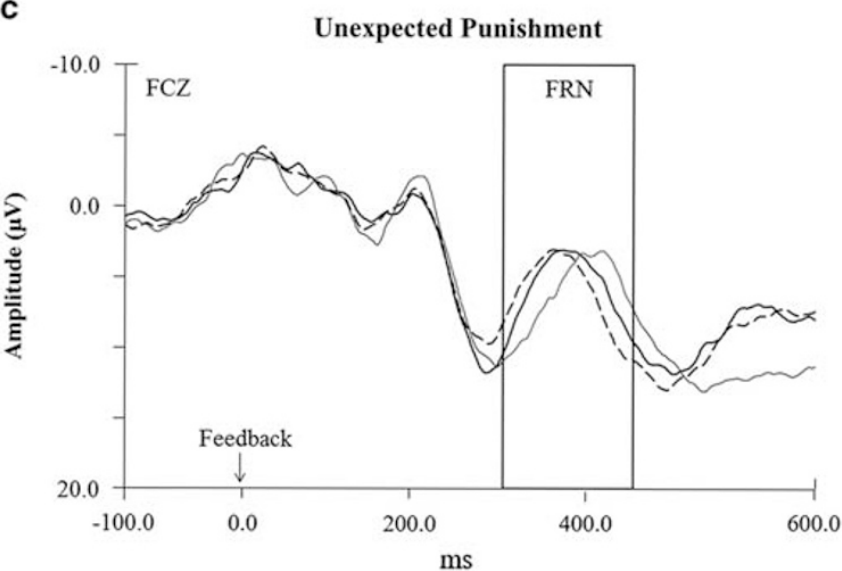

d

Difference Waveform (UP-UR)
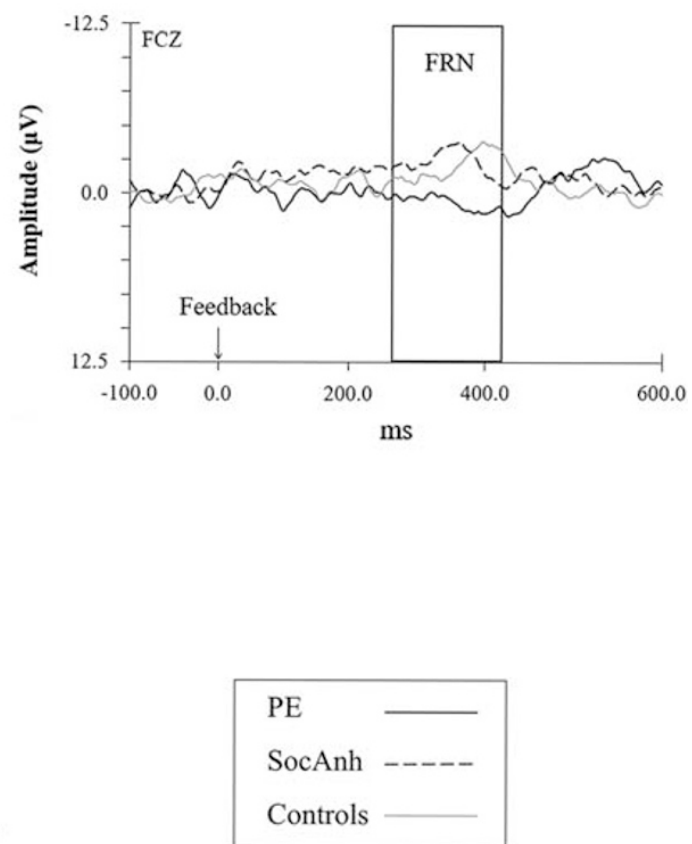

Figure 2 The psychotic experiences (PE) group showed similar FRNs to punishment and reward, with a numerically larger feedback-related negativity (FRN) to reward than punishment (225-325 ms post-stimulus) elicited at the FCZ electrode for each of the groups. Note that following common eventrelated potential (ERP) research convention and because the FRN is a negative deflection, negative values are plotted upwards. (a) Grand average waveforms comparing the unexpected reward (UR) and unexpected punishment (UP) conditions for each of the groups. (b) Grand average waveform for the unexpected reward (UR) condition. (c) Grand average waveform for the unexpected punishment (UP). (d) Grand average difference waveform for Reward Sensitivity Differences Score (UP FRN - UR FRN).

results could help us better understand the nature of psychosis risk and also might prove useful in better identifying people at risk for psychotic disorders.

In terms of their behavior, individuals in the PE group exhibited greater sensitivity to learning from unexpected reward than from unexpected punishment, as reaction times on trials after unexpected reward were significantly faster than after unexpected punishment. Previous research using this version of the RLT has found consistent evidence that increased striatal dopamine is associated with increased sensitivity to learning from unexpected reward (van der Schaaf et al, 2014). Similarly, other behavioral research also has found that increased dopamine in the striatum is related to increased sensitivity to learning from rewards (Frank et al, 
Table 3 P3a Amplitude Means (and SD) for Unexpected Reward and Unexpected Punishment for each of the Groups for FCZ and CZ Electrodes

\begin{tabular}{lccc}
\hline Condition & \multicolumn{3}{c}{ Group } \\
\cline { 2 - 4 } & PE & SocAnh & Control \\
\hline Unexpected punishment (UP) & $8.18(5.89)$ & $9.30(8.09)$ & $10.90(6.10)$ \\
Unexpected reward (UR) & $11.15(10.76)$ & $8.89(6.17)$ & $9.56(6.31)$ \\
Reward Sensitivity Difference & $-2.75(8.07)^{*}$ & $0.406 .29)$ & $1.34(7.47)$ \\
Score (UP - UR) & & & \\
\hline
\end{tabular}

Abbreviations: PE, psychotic experiences group; SocAnh, Social

Anhedonia group.

Asterisk indicates that the PE group differed significantly from the other two groups.

2004). Hence, the current evidence of PE being associated with greater sensitivity to learning from unexpected reward than from unexpected punishment is consistent with increased dopamine in the striatum in people with psychosis risk (Karcher et al, 2015b).

In addition to $\mathrm{PE}$ being associated with increased behavioral sensitivity to reward, while the control and SocAnh groups showed a larger FRN to punishment than reward, the PE group showed similar FRNs to punishment and reward, with a numerically larger FRN to reward than punishment. Moreover, whereas the groups did not differ in their FRN responses to unexpected punishment, the FRN to unexpected reward was larger in the $\mathrm{PE}$ group than in the other two groups. A relatively increased neural response for unexpected reward in psychosis risk is consistent with previous evidence on striatal dopamine and the FRN. Dopamine agonists have been found to increase the FRN for unexpected reward (Santesso et al, 2009). The FRN has been found to increase for salient stimuli (Pfabigan et al, 2015), and there is evidence that increased striatal dopamine enhances processing of rewarding information (Collins and Frank, 2014). There is also evidence that the FRN to reward increases with increased reward sensitivity (Smillie et al, 2011). Hence, the current FRN results in the $\mathrm{PE}$ group are consistent with an increase of striatal dopamine in psychosis risk. Moreover, in additional analyses, results for the P3a were similar to the results for the FRN, with larger neural responses to reward than to punishment in the PE group. Therefore, in the current study multiple behavioral and neural indicators were consistent with the possibility that psychosis risk is associated with increased striatal dopamine. The current results suggest that behavioral and electrophysiological measures have the potential to successfully identify people at risk for psychosis by providing evidence of dopamine dysregulation (Wagshal et al, 2012; Weickert et al, 2010). Furthermore, it should be noted that the results of the current study differ from the results of a previous study examining reversal learning (von Borries et al, 2013), which found that the FRN varied as a function of valence, but not expectancy (ie, in that study there was not a significant difference in the FRN for expected $v s$ unexpected trials). Compared with our study, that previous study had smaller runs of consecutive expected trials, which may have reduced the difference between expected and unexpected trials.

One issue for future psychosis risk research is to continue to examine the nature of striatal dysfunction observed in the current study. For instance, it is possible that the FRN is the result of two functionally and empirically distinct neural responses that overlap in time, one that is more reflective of feedback salience, reflecting the $\mathrm{N} 2$, and a second that is more reflective of positive valence, labeled positivity to rewards (RewP; Holroyd and Coles, 2002; Proudfit, 2015). The current ERP results for the PE group presumably reflect increased salience to reward feedback and an increased N2, rather than an altered RewP. Future research could attempt to separately assess these two FRN-related components, possibly using principal components analysis, and examine which one is associated with psychosis risk. Another interpretation of the altered FRN in the PE group is a lack of sensitivity to outcome valence, given that the PE group did not exhibit a difference in the FRN for reward $v s$ punishment trials. Although the FRN results could suggest a lack of outcome sensitivity in the $\mathrm{PE}$ group, there was other evidence (behavioral and $\mathrm{P} 3 \mathrm{a}$ ) that this group was more sensitive to reward $v s$ punishment outcome information than the other groups, which perhaps could also explain the FRN results in the PE group. Hence, one issue for future research would be to further examine whether the FRN results in the $\mathrm{PE}$ group reflect a lack of sensitivity to outcome valence or an increased sensitivity to reward $v s$ punishment information. In addition, future research could examine performance on the RLT in people with psychosis risk using fMRI to examine functioning of the striatum and other potentially relevant brain regions (eg, ACC) during this task. Also, one limitation of the study is that we did not assess medication usage. However, in a recent study utilizing the same recruitment method, none of the individuals in the PE had a history of antipsychotic medication usage. Therefore, it seems reasonable to expect that none, or at the most perhaps a very small number, of the people in the PE group in the sample for the current manuscript had ever previously taken antipsychotic medication.

In contrast to the $\mathrm{PE}$ group, the social anhedonia group did not differ from the control group in their behavioral performance or in their FRN for unexpected trials on the RLT, suggesting that some aspects of reward and punishment-based learning may to some extent be intact in people with extremely elevated social anhedonia who are at increased risk for schizophrenia-spectrum disorders (Karcher et al, 2015b; Padrao et al, 2013). Future research should continue to examine whether some particular aspect of reward and punishment-based learning, perhaps reward anticipation (Dowd and Barch, 2012; Juckel et al, 2006), is associated with anhedonia in at risk groups.

\section{FUNDING AND DISCLOSURE}

Dr Bartholow has received research funding from the National Institutes of Health (NIH) and has reviewed grant proposals for the NIH and other agencies; has served as Associate Editor for academic journals; and has given academic lectures at numerous universities and medical centers. Dr Martin has consulted for Evidera and provided 
clinical care at McLean Hospital/Harvard Medical School. She received support through a Hellman Fellowship given by the University of California and performed grant reviews for NSF. Dr Kerns has received grant funding from National Institutes of Mental Health (NIMH) and has reviewed grant proposals for NIH. The authors declare no conflict of interest.

\section{REFERENCES}

Baker TE, Holroyd CB (2011). Dissociated roles of the anterior cingulate cortex in reward and conflict processing as revealed by the feedback error-related negativity and N200. Biol Psychol 87: 25-34.

Bates DM (2010). lme4: Mixed-effects modeling with R. Retrieved from http://lme4.r-forge.r-project.org/lMMwR/lrgprt.pdf.

Becker MP, Nitsch AM, Miltner WH, Straube T (2014). A singletrial estimation of the feedback-related negativity and its relation to BOLD responses in a time-estimation task. J Neurosci 34: 3005-3012.

Bismark AW, Hajcak G, Whitworth NM, Allen JJ (2013). The role of outcome expectations in the generation of the feedback-related negativity. Psychophysiology 50: 125-133.

Carlson JM, Foti D, Mujica-Parodi LR, Harmon-Jones E, Hajcak G (2011). Ventral striatal and medial prefrontal BOLD activation is correlated with reward-related electrocortical activity: a combined ERP and fMRI study. Neuroimage 57: $1608-1616$.

Chapman LJ, Chapman JP, Kwapil TR, Eckblad M, Zinser MC (1994). Putatively psychosis-prone subjects 10 years later. J Abnorm Psychol 103: 171-183.

Chapman LJ, Chapman JP, Raulin ML (1978). Body-image aberration in schizophrenia. J Abnorm Psychol 87: 399-407.

Collins AG, Frank MJ (2014). Opponent actor learning (OpAL): modeling interactive effects of striatal dopamine on reinforcement learning and choice incentive. Psychol Rev 121: 337-366.

Cools R, Frank MJ, Gibbs SE, Miyakawa A, Jagust W, D’Esposito M (2009). Striatal dopamine predicts outcome-specific reversal learning and its sensitivity to dopaminergic drug administration. J Neurosci 29: 1538-1543.

Debbané M, Eliez S, Badoud D, Conus P, Flückiger R, SchultzeLutter F (2015). Developing psychosis and its risk states through the lens of schizotypy. Schizophr Bull 41: S396-S407.

Demjaha A, Murray RM, McGuire PK, Kapur S, Howes OD (2012). Dopamine synthesis capacity in patients with treatment-resistant schizophrenia. Am J Psychiatry 169: 1203-1210.

Dowd EC, Barch DM (2012). Pavlovian reward prediction and receipt in schizophrenia: relationship to anhedonia. PLOS ONE 7: e35622.

Eckblad M, Chapman LJ (1983). Magical ideation as an indicator of schizotypy. J Consult Clin Psychol 51: 215-225.

Eckblad M, Chapman LJ, Chapman JP, Mishlove M (1982). The revised social anhedonia scale. Unpublished test, University of Wisconsin, Madison, WI.

Evans DE, Maxfield ND, Van Rensburg KJ, Oliver JA, Jentink KG, Drobes DJ (2013). Nicotine deprivation influences P300 markers of cognitive control. Neuropsychopharmacology 38: $2525-2531$.

Frank MJ, Seeberger LC, O'Reilly RC (2004). By carrot or by stick: cognitive reinforcement learning in parkinsonism. Science 306: 1940-1943.

Gratton G (2007). Biosignal processing. In: Cacioppo J, Tassinary L, Berntson G (eds). Handbook of Psychophysiology. Cambridge University Press: New York. pp 900-923.
Holroyd CB, Coles MG (2002). The neural basis of human error processing: reinforcement learning, dopamine, and the errorrelated negativity. Psychol Rev 109: 679-709.

Holroyd CB, Nieuwenhuis S, Yeung N, Cohen JD (2003). Errors in reward prediction are reflected in the event-related brain potential. Neuroreport 14: 2481-2484.

Howes O, Kapur S (2009). The dopamine hypothesis of schizophrenia: Version III-The final common pathway. Schizophr Bull 35: 549-562.

Jennings JR, Wood CC (1976). The e-adjustment procedure for repeated-measures analyses of variance. Psychophysiology 76: 277-278.

Juckel G, Schlagenhauf F, Koslowski M, Wustenberg T, Villringer A, Knutson B et al (2006). Dysfunction of ventral striatal reward prediction in schizophrenia. Neuroimage 29: 409-416.

Karcher NR, Cicero D, Kerns JG (2015a). An experimental examination of the aberrant salience hypothesis using a salience manipulation and a behavioral magical thinking task. J Exp Psychopathol 6: 297-312.

Karcher NR, Martin EA, Kerns JG (2015b). Examining associations between psychosis risk, social anhedonia, and performance of striatum-related behavioral tasks. J Abnorm Psychol 124: 507-518.

Kaymaz N, Drukker M, Lieb R, Wittchen HU, Werbeloff N, Weiser $M$ et al (2012). Do subthreshold psychotic experiences predict clinical outcomes in unselected non-help-seeking population-based samples? A systematic review and metaanalysis, enriched with new results. Psychol Med 42: 2239-2253.

Luck SJ (2005). An Introduction to the Event-Related Potential Technique. MIT Press: Cambridge.

Miller TJ, McGlashan TH, Rosen JL, Cadenhead K, Cannon T, Ventura J et al (2003). Prodromal assessment with the structured interview for prodromal syndromes and the scale of prodromal symptoms: predictive validity, interrater reliability, and training to reliability. Schizophr Bull 29: 703-715.

Nieuwenhuis S, Slagter HA, von Geusau NJ, Heslenfeld DJ, Holroyd CB (2005). Knowing good from bad: differential activation of human cortical areas by positive and negative outcomes. Eur J Neurosci 21: 3161-3168.

Padrao G, Mallorqui A, Cucurell D, Marco-Pallares J, RodriguezFornells A (2013). Neurophysiological differences in reward processing in anhedonics. Cogn Affect Behav Neurosci 13: $102-115$.

Page-Gould E. Multilevel modeling. Cacioppo JT, Tassinary L, Berntson G (eds). The Handbook of Psychophysiology (in press) 4th edn. Cambridge University Press: New York.

Pfabigan DM, Seidel EM, Paul K, Grahl A, Sailer U, Lanzenberger R et al (2015). Context-sensitivity of the feedback-related negativity for zero-value feedback outcomes. Biol Psychol 104: 184-192.

Polich J (2007). Updating P300: An integrative theory of P3a and P3b. Clin Neurophysiol 118: 2128-2148.

Proudfit GH (2015). The reward positivity: From basic research on reward to a biomarker for depression. Psychophysiology 52: 449-459.

Psychology Software Tools, Inc. (2006). E-prime v. 2.0. Pittsburg, PA.

Santesso DL, Evins AE, Frank MJ, Schetter EC, Bogdan R, Pizzagalli DA (2009). Single dose of a dopamine agonist impairs reinforcement learning in humans: evidence from event-related potentials and computational modeling of striatal-cortical function. Hum Brain Mapp 30: 1963-1976.

Smillie LD, Cooper AJ, Pickering AD (2011). Individual differences in reward-prediction-error: extraversion and feedback-related negativity. Soc Cogn Affect Neurosci 6: 646-652.

van der Schaaf ME, van Schouwenburg MR, Geurts DE, Schellekens AF, Buitelaar JK, Verkes RJ et al (2014). Establishing the dopamine dependency of human striatal signals during reward and punishment reversal learning. Cereb Cortex 24: 633-642. 
von Borries AK, Verkes RJ, Bulten BH, Cools R, de Bruijn ER (2013). Feedback-related negativity codes outcome valence, but not outcome expectancy, during reversal learning. Cogn Affect Behav Neurosci 13: 737-746.

Wagshal D, Knowlton B, Cohen J, Poldrack R, Bookheimer S, Bilder $\mathrm{R}$ et al (2012). Deficits in probabilistic classification learning and liability for schizophrenia. Psychiatry Res 200: $167-172$.
Walsh MM, Anderson JR (2012). Learning from experience: eventrelated potential correlates of reward processing, neural adaptation, and behavioral choice. Neurosci Biobehav Rev 36: 1870-1884.

Weickert TW, Goldberg TE, Egan MF, Apud JA, Meeter M, Myers CE et al (2010). Relative risk of probabilistic category learning deficits in patients with schizophrenia and their siblings. Biol Psychiatry 67: 948-955.

Supplementary Information accompanies the paper on the Neuropsychopharmacology website (http://www.nature.com/npp) 